\title{
Directionality of Association between Panic Disorder and Depression in a Nationwide Epidemiological Survey
}

\author{
Jungmin Woo, ${ }^{1}$ Jin Pyo Hong, ${ }^{2}$ Seong-Jin Cho, ${ }^{3}$ Jun-Young Lee, ${ }^{4}$ Hong Jin Joen, ${ }^{2}$ Byung-Soo Kim, ${ }^{1}$ and Sung Man Chang ${ }^{1}$ \\ ${ }^{1}$ Department of Psychiatry, School of Medicine, Kyungpook National University, Daegu, Korea. \\ 2Department of Psychiatry, Samsung Medical Center, Sungkyunkwan University School of Medicine, Seoul, Korea. \\ ${ }^{3}$ Department of Psychiatry, Gachon Medical School, Gachon University of Medicine and Science, Incheon, Korea. \\ ${ }^{4}$ Department of Neuropsychiatry, Seoul Metropolitan Boramae Medical Center, Seoul, Korea.
}

Background: The comorbidity of panic disorder (PD) and major depressive disorder (MDD) is common in both clinical and general settings. Although this comorbidity is very prevalent, few researchers and clinicians have pay attention to this. Moreover, we may not consider the association of them since the symptomatic and biological profiles of these two disorders are quite different. In this study, we analyze the association between PD and MDD, with respect to the time interval, and verify the directionality.

Methods: Data from three national representative surveys were pooled, and the age of onset (AOO) of PD and MDD was analyzed. These three surveys investigated the prevalence of psychiatric disorders based on the DSM-IV-TR in Korean adults aged 18 years and older. Subjects were selected by multistage, cluster sampling of 18,807 adults across 12 catchment areas. We used the Kaplan-Meier method to estimate separate survival functions, using the age of onset (AOO) of MDD and PD as the outcome. For the survival analysis, in which MDD/PD was the outcome, the AOO of MDD/PD was the endpoint for patients who experienced an episode of MDD/PD. To understand the temporal effects of previous disorders, we used a Cox proportional hazard model to estimate HRs for the onset of MDD/PD with the comorbid disorder as a time-dependent covariate.
Lifetime prevalence of panic disorder and major depressive disorder as comorbidities. Unweighted $\mathbf{N}$ (Weighted \%)

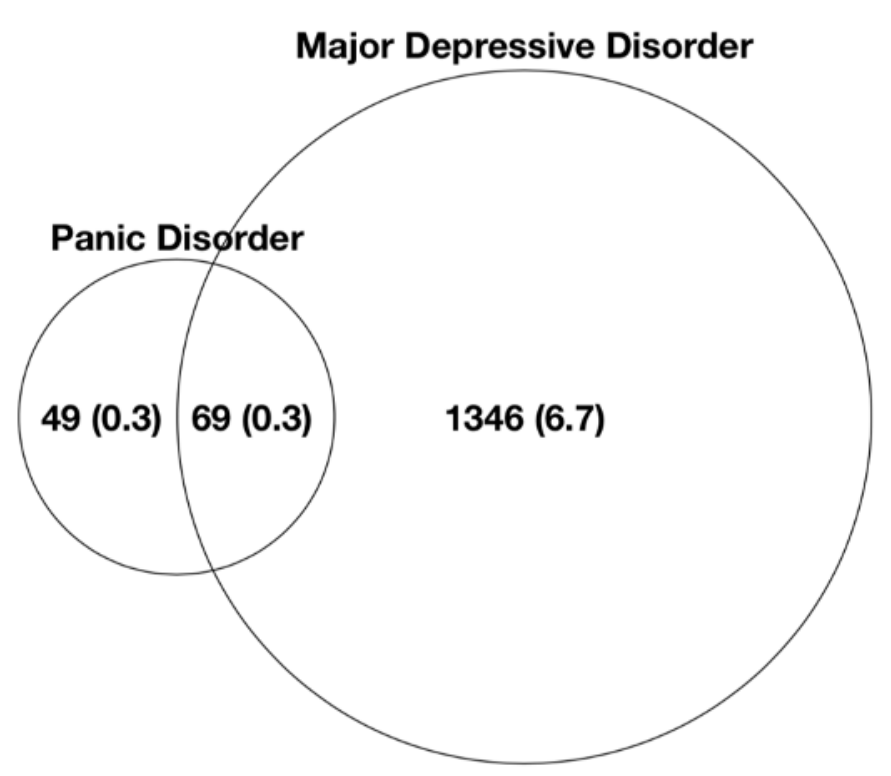

Results: PD elevated the risk of subsequent MDD by 1.5 times $(p=$ 0.004 ), whereas MDD elevated the risk of subsequent PD by 3.8 times $(p=0.001)$. The effect of such an elevation risk was significant for up to 2 years (PD to MDD: HR of same year $=1.626, p=0.035$; HR of 1-2 years $=2.248, p=0.015 ;$ MDD to PD: $H R$ of same year $=4.339, p$ $<0.001$; HR of $1-2$ years $=7.758, p<0.001$ ).

Conclusion: The results revealed a bidirectional relationship between MDD and PD. Each disease represents a risk of a subsequent occurrence of the other, which lasts for a considerable duration.

Cumulative occurrence of PD over time in patients with and without MDD / Hazard ratios for the risk of PD associated with MDD

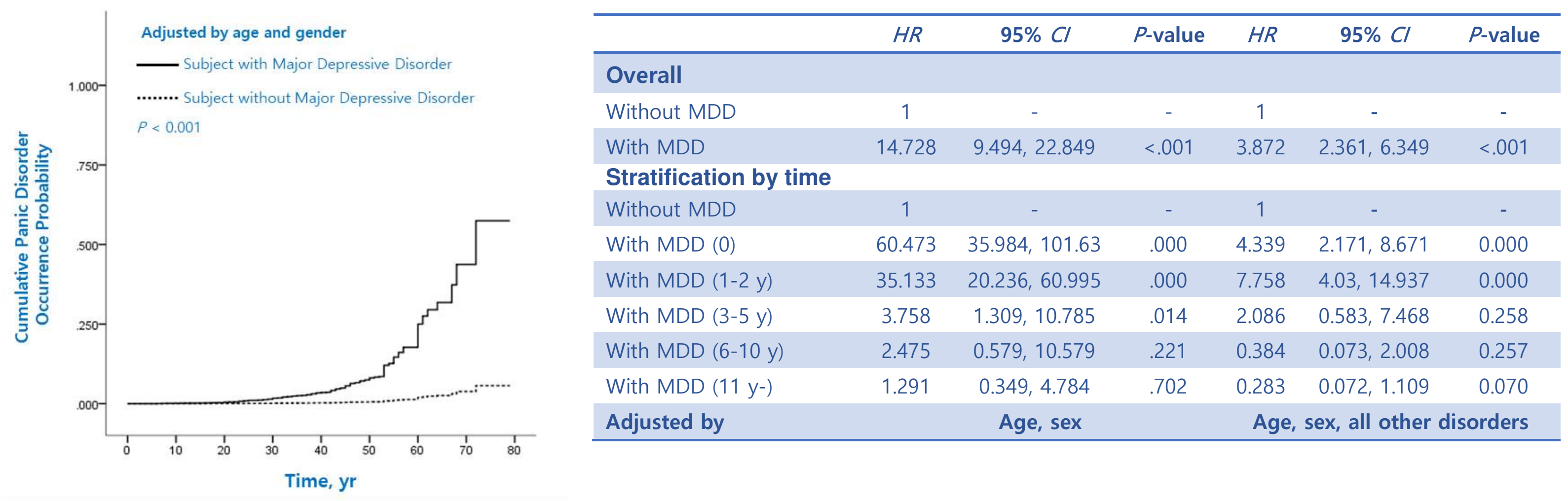

Cumulative occurrence of MDD over time in patients with and without PD / Hazard ratios for the risk of MDD associated with PD

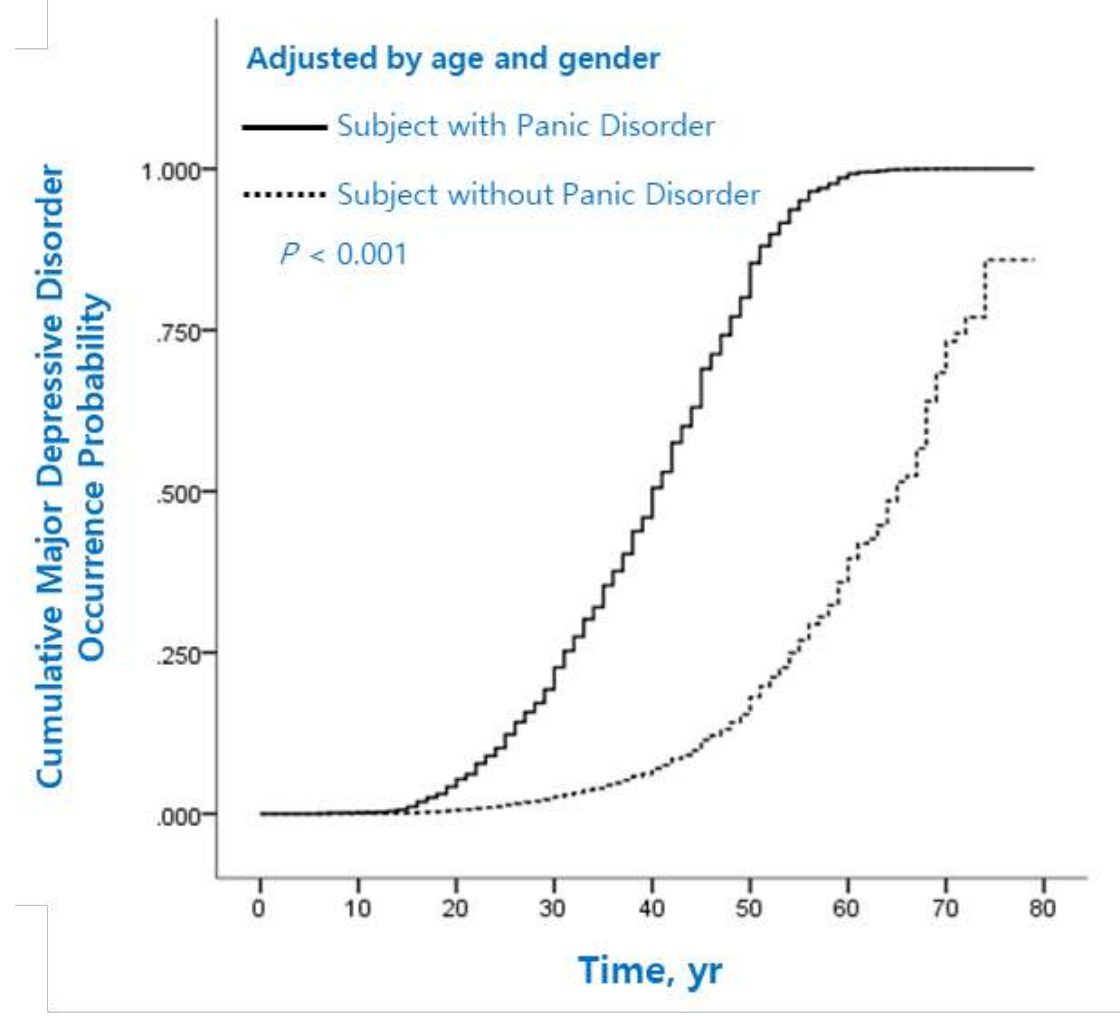

\begin{tabular}{|c|c|c|c|c|c|c|}
\hline & $H R$ & $95 \% \mathrm{Cl}$ & $P$-value & $H R$ & $95 \% \mathrm{Cl}$ & $P$-value \\
\hline \multicolumn{7}{|l|}{ Overall } \\
\hline Without PD & 1 & - & - & - & - & - \\
\hline With PD & 9.638 & $7.203,12.896$ & $<0.001$ & 1.518 & $1.147,2.009$ & 0.004 \\
\hline \multicolumn{7}{|c|}{ Stratification by time } \\
\hline Without PD & 1 & - & - & 1 & - & - \\
\hline With PD (0) & 19.244 & $14.47,25.591$ & $<0.001$ & 1.626 & $1.034,2.556$ & 0.035 \\
\hline With PD (1-2 y) & 12.680 & $6.24,25.763$ & $<0.001$ & 2.248 & $1.167,4.328$ & 0.015 \\
\hline With PD (3-5 y) & 4.439 & $1.782,11.055$ & $<0.001$ & 1.008 & $0.587,1.731$ & 0.977 \\
\hline With PD (6-10 y) & 2.699 & $0.855,8.52$ & 0.091 & 1.149 & $0.574,2.298$ & 0.695 \\
\hline With PD (11 y-) & 7.156 & $3.294,15.546$ & $<0.001$ & 1.032 & $0.568,1.876$ & 0.917 \\
\hline Adjusted by & \multicolumn{3}{|c|}{ Age, sex } & \multicolumn{3}{|c|}{ Aae sex all other disorders } \\
\hline
\end{tabular}

